not been sufficiently studied. Sectional analyses were conducted among menopaused employees $(n=486)$, participating in a longitudinal study (the Pró-Saúde Study). Data on insomnia complaints (IC), menopause characteristics, self-report of physical morbidity medical diagnosis, common mental disorders (CMD), social support and stressful life events (SLE) were collected. Insomnia complaints were analysed as polytomic outcome (frequent, occasional and absent), and crude and adjusted ORs were calculated by multinomial logistic regression. Prevalences of frequent and occasional IC among women in menopause were $25.7 \%$ and $32.7 \%$, respectively. Menopause characteristics were not associated with IC. Presence of CMD (GHQ-12) was strongly associated with frequent IC $[\mathrm{OR}=6.14$ (95\% CI 3.24 to 11.65$)]$. For occasional IC, values were lower $[\mathrm{OR}=2.98$ (95\% CI 1.59 to 5.57)], but still significant. All social support dimensions were associated with occasional IC, after adjustment, except for the intermediate tercile of total social support. The social support dimension associated with frequent IC after adjustment was "to have low total social support" [OR=2.46 (95\% CI 1.33 to 4.56$)$ ], "to have low emotional support/information" [OR=2.31 (CI 95\% 1.27 to 4.19$)$ ], and "to have low affective support/positive social interaction" [OR=2.21 (CI 95\% 1.22 to 4.02)]. Our results warn of an important association among insomnia complaints and $\mathrm{CMD}$ and social support. These psychosocial factors can contribute to the onset of primary insomnia, a symptom that in general is not taken into consideration when the patient seeks for medical help.

\section{P1-503 MENOPAUSE AND INSOMNIA IN THE PRÓ-SAÚDE STUDY: ASSESSING THE ROLE OF PSYCHOSOCIAL, SOCIO-ECONOMIC AND DEMOGRAPHIC FACTORS}

doi:10.1136/jech.2011.142976g.91

1J R Robaina, ${ }^{*} \mathrm{C}$ de S Lopes, ${ }^{2} \mathrm{~L}$ Rotenberg. ${ }^{1}$ Universidade do Estado do Rio de Janeiro, Rio de Janeiro, Rio de Janeiro, Brazil; ${ }^{2}$ Fundação Oswaldo Cruz, Rio de Janeiro, Rio de Janeiro, Brazil

Studies show that insomnia in menopause may be associated with psychological symptoms, which would reflect its association with depression and high levels of tension and stress. To estimate the prevalence of insomnia and to assess its association with menopausal status in a population of women, a cross-sectional study was conducted among employees of a university in Rio de Janeiro, participating in a longitudinal study (the Pró-Saúde Study) $(n=2189)$, using a self-administered questionnaire to evaluate insomnia, menopausal status and other variables. Non-pregnant women who reported absence of menstruation were considered "in menopause". Insomnia was ranked as difficulty initiating sleep (DIS), difficulty maintaining sleep (DMS) and general insomnia complaints (GIC). Crude and adjusted ORs were calculated by multivariate logistic regression. Among women in menopause, prevalence of insomnia was $20.8 \%$ for difficulty initiating sleep, $21.8 \%$ for difficulty maintaining sleep, and $25.7 \%$ for general insomnia complaints. In the final model, adjusted by socio-economic and demographic variables, common mental disorder (CMD), stressful life event (SLE) and social support, all insomnia categories were associated with menopause. ORs were the following: for DIS $\mathrm{OR}=1.58$ (95\% CI 1.07 to 2.34 ); for DMS OR=1.61 (95\% CI 1.08 to 2.41 ); and for $\mathrm{GIC} \mathrm{OR}=1.68$ (95\% CI 1.15 to 2.43 ). These results are consistent with other studies, and show that menopause is significantly associated with the presence of insomnia, is independent of age, income, education, CMD, SLE and social support. The results of our study can help highlight the importance of managing/treating insomnia associated with menopause.

\section{P1-504 DIETARY INTAKE AND ADEQUACY TO NUTRITIONAL RECOMMENDATIONS IN PREGNANT WOMEN IN A MEDITERRANEAN AREA. INMA-VALENCIA COHORT}

doi:10.1136/jech.2011.142976g.92

${ }^{1} \mathrm{C}$ L Rodríguez-Bernal, ${ }^{2} \mathrm{R}$ Ramón, ${ }^{1} \mathrm{M}$ Murcia, ${ }^{2} \mathrm{~J}$ Quiles, ${ }^{3} \mathrm{~J}$ Vioque, ${ }^{3} \mathrm{E}$ Navarrete, ${ }^{2} \mathrm{M}$ Rebagliato, ${ }^{1} \mathrm{~F}$ Ballester. ${ }^{1}$ Centro Superior de Investigación en Salud Pública, Valencia, Spain; ${ }^{2}$ Dirección General de Salud Pública, Valencia, Spain; ${ }^{3}$ Universidad Miguel Hernández, Alicante, Spain

Introduction Maternal diet may be an important determinant of maternal and infant health outcomes. However, the nutritional status of pregnant women in the Spanish Mediterranean region has been scarcely studied.

Methods We studied 822 women from the INMA-Valencia cohort. Intakes from food and supplements in early pregnancy as well as adequacy to dietary recommendations were assessed according to maternal characteristics. Adequate intakes for food groups were estimated based on Spanish dietary guidelines. Intake inadequacy for nutrients was assessed using the Dietary Reference Intakes of the Institute of Medicine. Descriptive statistics of dietary intakes were reported. We contrasted the distribution and the adequacy of intake according to maternal characteristics by means of ANOVA, post-hoc tests and logistic regression.

Results Overall, more than $50 \%$ of the population studied had deficient intakes of cereals and legumes, carbohydrates, n-3 and n-6 fatty acids, and exceeded the recommendations for total fat intake. Prevalence of inadequacy for folate, iron and vitamin $\mathrm{E}$ from foods ranged from $99 \%$ to $70 \%$. Younger and less educated women had lower intakes of vegetables, proteins and n-3 fatty acids and higher intakes of trans-fatty acids along with a greater prevalence of inadequacy for micronutrients. Spanish women showed lower intakes of fruits and carbohydrates and higher intakes of proteins, total fat, saturated fatty acids, MUFA and n-3 fatty acids compared to their foreign counterparts.

Conclusion Women in the studied area have inadequate intakes of several nutrients relevant during pregnancy. Besides age and education, origin is an important determinant of dietary intake and adequacy.

\section{P1-505 CURRENT DIET VS DASH DIET ASSESSED BY FOOD FREQUENCY OUESTIONNAIRE USING FOOD GROUPS: AGREEMENT AMONG HYPERTENSIVE PATIENTS}

doi:10.1136/jech.2011.142976g.93

${ }^{1} \mathrm{~S}$ L Rossato, ${ }^{1} \mathrm{~F}$ Moselle, ${ }^{1} \mathrm{~F}$ Guizonni, ${ }^{1,2}$ L B Moreira, ${ }^{1,2} \mathrm{~S}$ C Fuchs. ${ }^{1}$ Universidade Federal do Rio Grande do Sul, Porto Alegre, Brazil; ${ }^{2}$ Hospital de Clinicas de Porto Alegre, Porto Alegre, Brazil

Introduction Dietary guidelines for hypertensive patients require methods able to identify food patterns and monitoring adherence, which could be based on a simplified Food Frequency Questionnaire (FFQ) for food groups.

Objective To develop a FFQ for food groups in order to easily recommend the DASH (Dietary Approach to Stop Hypertension) diet for hypertensive patients.

Methods A cross-sectional study was conducted among adults, aged 20 to 69 years, from a Hypertension clinic of a reference centre (Hospital de Clínicas de Porto Alegre), in southern Brazil. Data gathering using two sequential $24 \mathrm{~h}$ recalls (RA $24 \mathrm{~h}$ ) were adopted to assess dietary intake. A list of food groups was generated, based on the DASH diet adapted to the southern Brazil population. A pilot study, including 30 individuals, was conducted to test the FFO structure, consumption of food groups, two periods of recall, and pictures and wording for food items identification.

Results A sample of 127 hypertensive patients were interviewed at seven and 30 days, using a qualitative FFQ with 40 items aggregated 